\title{
Separation of Nanoparticles in Aqueous Multiphase Systems through Centrifugation
}

\section{Citation}

Akbulut, Ozge, Charles R. Mace, Ramses V. Martinez, Ashok A. Kumar, Zhihong Nie, Matthew R. Patton, and George M. Whitesides. 2012. Separation of Nanoparticles in Aqueous Multiphase Systems through Centrifugation. Nano Letters 12, no. 8: 4060-4064.

\section{Published Version}

doi:10.1021/nl301452x

\section{Permanent link}

http://nrs.harvard.edu/urn-3:HUL.InstRepos:11928050

\section{Terms of Use}

This article was downloaded from Harvard University's DASH repository, and is made available under the terms and conditions applicable to Open Access Policy Articles, as set forth at http:// nrs.harvard.edu/urn-3:HUL.InstRepos:dash.current.terms-of-use\#OAP

\section{Share Your Story}

The Harvard community has made this article openly available.

Please share how this access benefits you. Submit a story.

\section{Accessibility}




\section{Separation of Nanoparticles in Aqueous Multiphase Systems through Centrifugation}

Ozge Akbulut ${ }^{1}$, Charles R. Mace ${ }^{1}$, Ramses V. Martinez ${ }^{1}$, Ashok A. Kumar ${ }^{2}$, Zhihong Nie ${ }^{1}$, Matthew R. Patton ${ }^{1}$, and George M. Whitesides ${ }^{1,3,4^{*}}$

${ }^{1}$ Departments of Chemistry \& Chemical Biology, Harvard University, 12 Oxford Street, Cambridge, MA 02138, United States

${ }^{2}$ School of Engineering and Applied Sciences, Harvard University, 29 Oxford Street, Cambridge, MA 02138, United States

${ }^{3}$ Wyss Institute for Biologically Inspired Engineering, Harvard University, 60 Oxford Street, Cambridge, MA 02138, United States

${ }^{4}$ Kavli Institute for Bionano Science and Technology, Harvard University, 29 Oxford Street, Cambridge, MA 02138, United States

*Corresponding author email: gwhitesides@gmwgroup.harvard.edu 
Table of Contents Graphic

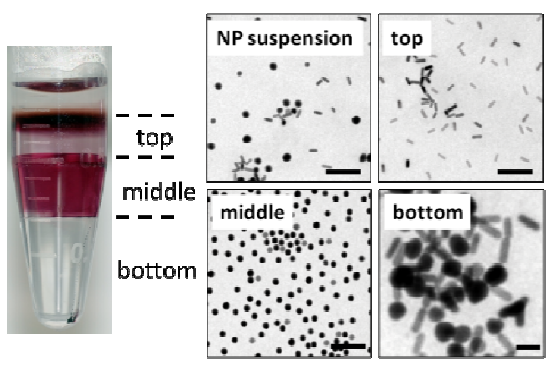




\begin{abstract}
This paper demonstrates the use of aqueous multiphase systems (MuPSs) as media for rate-zonal centrifugation to separate nanoparticles of different shapes and sizes. The properties of MuPSs do not change with time or during centrifugation; this stability facilitates sample collection after separation. A three-phase system demonstrates the separation of the reaction products (nanorods, nanospheres and large particles) of a synthesis of gold nanorods, and enriches the nanorods from $48 \%$ to $99 \%$ in less than ten minutes using a benchtop centrifuge.
\end{abstract}

Keywords: centrifugation, nanoparticles, separation, polymers, multiphase systems.

Exploiting the potential of nanoparticles in applications in self-assembly, electronics, diagnostics, and sensing ${ }^{1,2}$ may require monodisperse populations. Many syntheses of nanoparticles produce polydisperse mixtures, ${ }^{3,4}$ and techniques to enrich populations according to size, shape and/or composition are useful. This paper describes a new technique for separating gold nanoparticles according to their rate of migration through a viscous medium under centrifugal force.

Gold nanorods are potentially useful in diagnostic applications due to their unique optical properties such as tunable plasmonic fields. ${ }^{5,6}$ They can support a longitudinal surface plasmon with a strong extinction peak in the red end — the visible or near-infrared regions - of the spectrum; this characteristic suggests the use of gold nanorods as labels in biological imaging. ${ }^{7}$ The resonant wavelength of nanorods depends on their size and aspect ratio. ${ }^{8}$ Common preparations of gold nanorods - for example, those generated by the seed-mediated synthesisproduce a variety of shapes. ${ }^{8}$ Spheres, rather than rods, may comprise $5-20 \%(\mathrm{w} / \mathrm{w})$ of the gold 
nanoparticles formed in a representative synthesis. ${ }^{9}$ To establish the relationship between shape and optical properties of gold nanorods, and to examine the use of nanorods in technological applications, it would be convenient to have sub-populations of a mixture produced by a reaction easily accessible.

Methods for Separation of Nanorods. Sedimentation by centrifugation in the solvent used for the reaction (e.g., water or toluene) is a straightforward method to collect the reaction products generated in syntheses of gold nanorods. ${ }^{10}$ Multiple rounds of centrifugation are, however, usually necessary to separate by-products from rods. ${ }^{11,12}$ Other techniques used to separate monodisperse nanorods from mixtures are either time-consuming (e.g., size exclusion chromatography) ${ }^{13}$ or require secondary chemical modification of the nanoparticles (e.g., gel electrophoresis). ${ }^{14}$

The hydrodynamic behavior of particles depends upon their shape and size (Supporting Information, eqn.'s 1-4). Geometric differences can be used to sort particles dynamically in a medium that has a lower density than the density of the particles (e.g., $\rho_{\mathrm{Au}}=19.3 \mathrm{~g} / \mathrm{cm}^{3}$, and $\rho_{\text {media }}=1.0-1.4 \mathrm{~g} / \mathrm{cm}^{3}$ for aqueous solutions of sucrose). Viscosity amplifies the spatial separation between two particles with differences in hydrodynamic behavior.

Rate-zonal centrifugation uses the differences in hydrodynamic behavior to separate objects. ${ }^{15-20}$ In this technique, the medium of separation consists of zones of different viscosity. These zones are prepared by layering solutions of a single chemical in a common medium (e.g., solutions of Ficoll or sucrose in water) at different concentrations. The use of multiple zones, rather than a single one, is intended to facilitate sample collection and improve enrichment in hydrodynamic separation by localizing bands of nanoparticles to narrow regions. The boundaries between the layers of different viscosities are, however, not thermodynamically stable; they are 
disrupted easily by convection created during acceleration/deceleration of the centrifuge, and by diffusion of boundaries during centrifugation. These instabilities make it more difficult to collect separated populations cleanly. Separation by rate-zonal centrifugation, therefore, could benefit from a medium of centrifugation with sharp, well-defined and stable interfaces between zones of different density and/or viscosity.

The Use of Aqueous Multiphase Systems (MuPSs) as Media of Separation for Ratezonal Centrifugation. This paper describes a new approach to size- and shape-dependent separation of nanoparticles through rate-zonal centrifugation using MuPSs as separation media. We use this method to separate the reaction product (nanorods) and by-products (nanospheres and bigger particles) of a synthesis of gold nanorods.

Aqueous MuPSs are phase-separated mixtures of water-soluble polymers and/or surfactants. ${ }^{21}$ Upon separation in a gravitational field (slow) or through centrifugation (more rapid), the phases of MuPSs order according to their densities. These phases are characterized by different physical properties, such as (importantly for rate-zonal separations) viscosity.

The physical properties of the phases of a MuPS (e.g., viscosity, refractive index, ionic strength) do not necessarily correlate with density. For example, a three-phase MuPS composed of Brij 35 (a nonionic poly(ethylene oxide)-based surfactant), poly(2-ethyl-2-oxazoline) (PEOZ), and Ficoll (a polysucrose) has a highly viscous middle phase bordered by phases with lower viscosities (cf., Table 1). The ability to design and generate stacked, distinct fluid phases based on a common solvent (e.g., water), with viscosities chosen to give the best results in hydrodynamic separation, is the basis of the method of separating nanoparticles described here.

We have previously described the separation of objects based on their density by localization at the interfaces between the phases of MuPSs; this method can be described as 
"equilibrium density separation". ${ }^{21}$ Here, we use rate-zonal centrifugation through the phases of a MuPS, which have different viscosities, to separate objects with identical densities but with different hydrodynamic behaviors.

Compared to layered but miscible media, MuPSs offer five advantages: (i) the phases (i.e., the layers) of MuPSs are thermodynamically stable; (ii) the sharp interfaces between the phases facilitate the collection of samples after separation; (iii) the viscosities of each phase can be controlled independently of density; (iv) the MuPSs can be prepared in advance of use and stored; and (v) the MuPSs reform readily by centrifugation if disrupted. Isolating objects in different zones, where each zone is at equilibrium and separated by an interface, simplifies the recovery of enriched species.

MuPSs are versatile and easy to tune for specific separations. ${ }^{21}$ In layered miscible media, viscosity and density of each zone correlate closely and cannot be decoupled from each other. In MuPSs, it is possible to access a range of viscosities for a given density by using different combinations (e.g., different chemicals, or different molecular weights of the same polymer) of solutes that phase separate. (cf., Supporting Information, Table S1 and Table S2) Due to this tunability and inherent stability, MuPSs offer a valuable addition to the tools that can be used in hydrodynamic separations.

Synthesis of Nanoparticles. We used a seeded-growth method developed by Nikoobakht and El-Sayed, ${ }^{22}$ with minor modifications, to synthesize gold nanoparticles with an aspect ratio of approximately $4: 1$. It is possible to produce nanorods with a higher aspect ratio $(18: 1),{ }^{23}$ but a smaller aspect ratio better demonstrates the sensitivity of our approach to hydrodynamic separation of objects based on shape; the difference in sedimentation rates between a sphere and a rod increases in proportion to the aspect ratio (Supporting Information, eqn's 3 and 4). 
We concentrated as-synthesized nanoparticles produced in this method 20 -fold by centrifugation of the reaction medium at $16,000 \mathrm{~g}$ for ten minutes. The concentrated solution contained $48 \%$ rods and $52 \%$ spheres. The length of the rods was in the range of $32-355 \mathrm{~nm}$ and the thickness of rods was in the range of 8-125 nm; the diameter of spheres was in the range of 18-265 nm. We refer to this concentrated solution as "the suspension of nanoparticles".

Characterization of Populations of Nanoparticles. UV-Vis spectroscopy allowed the characterization of the populations of nanoparticles, both before and after separation, by quantifying the plasmonic absorption bands of their solutions. The wavelengths absorbed by gold nanoparticles depend on their size and shape. ${ }^{24}$ For these wavelengths $(400-1100 \mathrm{~nm})$, the polymers and surfactants of the MuPSs we used in this work are transparent and do not contribute to the measured absorbance. Transmission electron microscopy (TEM) on these samples characterized the size and shape of the nanoparticles of each layer, and allowed statistical analysis of the distribution of sizes and shapes of the isolated population. We used $n>$ 1000 particles for each population.

Selection of Parameters and Medium of Separation. The speed and quality of separation of two objects by rate-zonal centrifugation depends, in general, on the speed of centrifugation and the variables that determine the sedimentation coefficients of the objects (eqn.'s 1 and 2 in Supporting Information). Additional considerations exist when working with MuPSs because of the existence of multiple phases and interfaces; appropriate centrifugation parameters must be chosen to prevent the interfaces from trapping nanoparticles (eqn. 8 in Supporting Information).

Finally, the nanoparticles must be compatible with all the phases of the MuPS used for separation. For our application, we selected a MuPS that was compatible with cetyl 
trimethylammonium bromide (CTAB)-stabilized gold nanoparticles. We eliminated from consideration the systems comprising charged polymers and surfactants (e.g., poly(methacrylic acid), polyallylamine, poly(acrylic acid), poly(diallyldimethyl ammonium chloride), polyethyleneimine, and 3-[(3-cholamidopropyl)dimethylammonio]-1-propanesulfonate). Upon interaction with CTAB-stabilized gold nanoparticles, these systems formed gel-like structures, and precipitated the nanoparticles from the solution (data not shown). Non-ionic chemicals such as dextran, Ficoll (a polysucrose), poly(ethylene glycol) (PEG), poly(2-ethyl-2-oxazoline) (PEOZ), poly(vinyl alcohol) (PVA), Brij 35, and Pluronic F68 (a poly(ethylene oxide)poly(propylene oxide) copolymer-based surfactant) were compatible with nanoparticles.

The large library of aqueous polymers and surfactants that form MuPSs offers a wide range of systems with different viscosities and densities that can be used as media in separations. ${ }^{21}$ We investigated three-phase systems for this study to capture three main populations of the synthesis of nanorods that we use: small nanorods, small nanospheres, and bigger particles.

Determining the Viscosity of the Top Phase for Separation of Nanoparticles. We designed a MuPS with a top phase (lowest density) that had sufficiently high viscosity to delay the penetration of nanorods into the MuPS until the nanospheres, which have larger sedimentation velocities, migrated into the middle phase.

A solution containing a high percentage of nanorods should appear brown, while a solution containing mostly nanospheres should appear pink/red. ${ }^{3}$ We evaluated the separation of these species by observing the formation of a narrow brown band of nanorods on top of a singlephase polymer system and the sedimentation of this band into the medium (i.e., a rate-dependent separation of nanoparticles in a homogeneous medium). For this evaluation, we first examined 
dilutions of solutions of PEOZ (MW=200,000 g/mol). When the concentration of PEOZ in a solution is varied from $4 \%(\mathrm{w} / \mathrm{v})$ to $35 \%(\mathrm{w} / \mathrm{v})$, the viscosity of the solution covers a wide range (from $4 \mathrm{cP}$ to $1100 \mathrm{cP}$, respectively) while its density increases by only 6\% (Supporting Information, Table S3).

We layered a suspension of nanoparticles $(100 \mu \mathrm{L})$ onto solutions of PEOZ $(1 \mathrm{~mL})$. We then sedimented the nanoparticles through these single-phase systems by centrifugation at $16,000 \mathrm{~g}$ for different intervals of time (two to eight minutes) to determine the relationship between the effectiveness of separation and the viscosity of the layer (Supporting Information, Figure S1).

Viscosities greater than $65 \mathrm{cP}$ were too high for the effective separation of nanorods and nanospheres into distinct populations within eight minutes (i.e., we did not observe the formation of separate bands). In a solution with a viscosity of $19 \mathrm{cP}$, the nanorods started to penetrate into the system in less than four minutes. (Supporting Information, Figure S1) Considering that the suspension of nanoparticles we use is polydisperse (i.e., we expect a continuum in penetration distance without clear boundaries between different sizes of particles), it is crucial for our method to delay the penetration of nanorods into the system until the band of nanorods is free of nanospheres. Based on these experiments, we concluded that a range for the viscosity of the top phase of a MuPS between 20 and $65 \mathrm{cP}$ would produce an optimal separation. This conclusion was also supported by our attempts to separate populations of nanoparticles in two different systems (i) a two-phase system composed of PEG ( $40 \%$ w/v), 1-O-octyl- $\beta$-D-glucopyranoside $(10 \% \mathrm{w} / \mathrm{v})$, with a low viscosity top phase (3.9 cP), and (ii) a three-phase system composed of PEG $(40 \% \mathrm{w} / \mathrm{v})$, dextran $(30 \% \mathrm{w} / \mathrm{v})$, and PEOZ (35\% w/v) with a high viscosity top phase (69 cP). In the former case, the nanoparticles penetrated into the system without forming bands. In 
the latter case, even the larger particles could not advance into the middle phase in less than ten minutes of centrifugation at 16,000g (Supporting Information, Figure S3).

Separation of Reaction Products of a Nanorod Synthesis. We prepared a series of three-phase MuPSs and measured the viscosity of each phase in order to evaluate their suitability as centrifugation media for enrichment of gold nanorods. The three phases of a MuPS should have viscosities that allow the three sub-populations of interest (i.e., nanorods, nanospheres, and larger particles) to be separated within ten minutes of centrifugation. The top phase should retain the nanorods, which have the smallest sedimentation coefficient of the three sub-populations, while allowing the other populations to pass through within this time. The middle phase should be viscous enough to capture nanospheres while allowing the larger particles to pass to the bottom phase. Of the MuPSs that we tested, we obtained the best enrichment of gold nanorods in a three-phase system composed of Brij $35(26 \% \mathrm{w} / \mathrm{v})$, PEOZ (30\% w/v) and Ficoll (35\% w/v) (Figure 1). The properties (density, $\rho$, and viscosity, $\eta$ ) of this system were: (i) $\rho_{\text {top }}=1.031 \mathrm{~g} / \mathrm{cm}^{3}$, $\eta_{\text {top }}=31 \mathrm{cP}$; (ii) $\rho_{\text {middle }}=1.045 \mathrm{~g} / \mathrm{cm}^{3}, \eta_{\text {middle }}=542 \mathrm{cP}$; and (iii) $\rho_{\text {bottom }}=1.112 \mathrm{~g} / \mathrm{cm}^{3}, \eta_{\text {bottom }}=$ $139 \mathrm{cP}$.

We layered the suspension of nanoparticles on this three-phase system and centrifuged these systems at $16,000 \mathrm{~g}$ for one to ten minutes. The effect of time on the penetration of particles into this MuPS was monitored by eye and quantified using spectroscopy and transmission electron microscopy (TEM). Figure 1 shows an example of penetration of nanoparticles into this MuPS. During centrifugation, the small nanorods were enriched in the top phase of the MuPS and formed a brown band. The penetration of small nanospheres into the 
Figure 1. The evolution of the penetration of nanoparticles into an aqueous three-phase system composed of Brij 35 (26\% w/v), PEOZ (30\% w/v) and Ficoll (35\% w/v) with time during centrifugation at $16,000 \mathrm{~g}$. The solvent of the suspension of nanoparticles (i.e., water) stayed as a clarified layer on top of the system, small nanorods (i.e., the desired product) penetrated slightly into the top phase, small nanospheres migrated to the middle phase, and large particles of both shapes sedimented to the bottom.

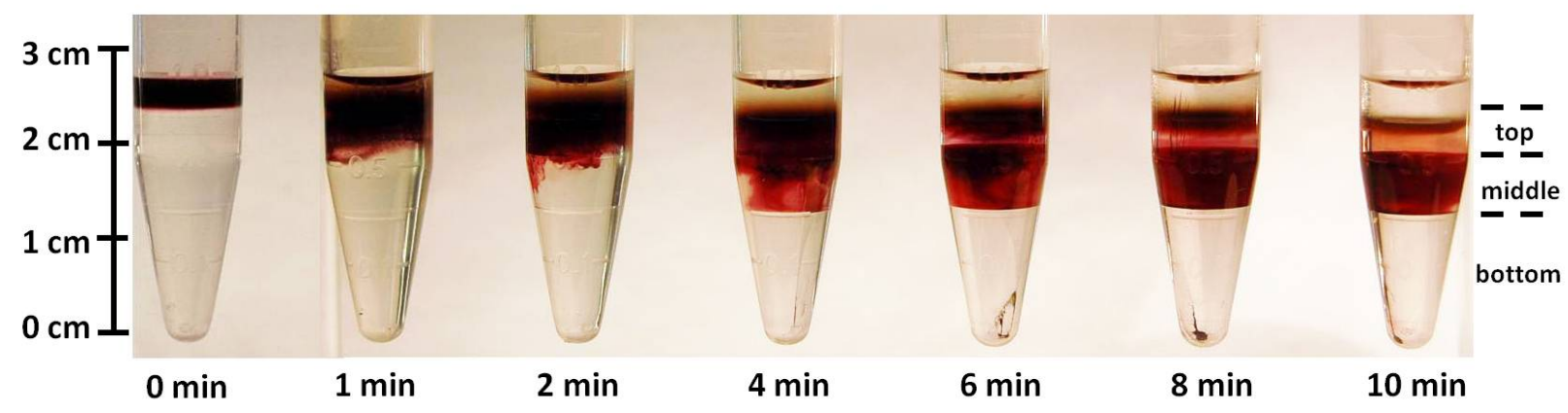


middle phase was visualized by the color change of this phase from transparent to red. After the complete migration of nanoparticles from the solution (i.e., suspension of nanoparticles) that was layered onto the MuPS, this solution appears as a transparent band at the top the MuPS. In this system, it took a $25-\mathrm{nm}$ diameter sphere three to four minutes at $16,000 \mathrm{~g}$ to migrate through the top phase, and to begin to penetrate the middle phase. A nanorod ( $l=36 \mathrm{~nm}, d=11 \mathrm{~nm})$ would sediment about four times more slowly than a sphere (Supporting Information, eqn.'s 3 and 4), hence requiring twelve to sixteen minutes to travel through the top phase of the MuPS under these conditions. Based on these estimates, we expect optimal separation to require between five to ten minutes of centrifugation.

We used a fixed angle benchtop centrifuge (VWR 1816) without temperature control to demonstrate simplicity of our method. We found reasonable agreement between the theoretical predictions and experimental results. The nanoparticles travel approximately three times more rapidly in the system that we use than predicted by theoretical calculations using eqn.'s $2-4$ (Supporting Information). We attribute this difference to the heating of the sample during centrifugation. This heating leads to a corresponding decrease in viscosity of the phases (the viscosity of the top phase drops from $31 \mathrm{cP}$ to $12 \mathrm{cP}$ with an increase from $22{ }^{\circ} \mathrm{C}$ to $28{ }^{\circ} \mathrm{C}$ after eight minutes of centrifugation at $16,000 \mathrm{~g}$ in the centrifuge that we use).

We quantified the efficiency of the separation of nanoparticles by an aqueous three-phase system using TEM and UV-Vis spectroscopy (Figure 2). In the top phase, nanorods were enriched to $99 \%$ (from $48 \%$ in suspension of nanoparticles) and the average length and thickness of the nanorods was $36 \pm 4 \mathrm{~nm}$ and $11 \pm 1 \mathrm{~nm}$, respectively. The amount of nanorods of similar size in other layers was less than $1 \%$; these values demonstrate that the method we use here is 
Figure 2. (a) Image of an aqueous three-phase system composed of Brij 35 (26\% w/v), PEOZ $(30 \% \mathrm{w} / \mathrm{v})$ and Ficoll $(35 \% \mathrm{w} / \mathrm{v})$ after sedimenting the reaction products of a gold nanoparticle synthesis for eight minutes at $16,000 \mathrm{~g}$. The properties of the phases of this system are: (i) $\rho_{\text {top }}=$ $1.031 \mathrm{~g} / \mathrm{cm}^{3}, \eta_{\text {top }}=30.8 \mathrm{cP}$; (ii) $\rho_{\text {middle }}=1.045 \mathrm{~g} / \mathrm{cm}^{3}, \eta_{\text {middle }}=541.9 \mathrm{cP}$; (iii) $\rho_{\text {bottom }}=1.112 \mathrm{~g} / \mathrm{cm}^{3}$, $\eta_{\text {top }}=139.0 \mathrm{cP}$. (b) TEM images of suspension of nanoparticles (suspension of NP) and samples collected from the layers as shown in (a). The scale bar in each image corresponds to $200 \mathrm{~nm}$.
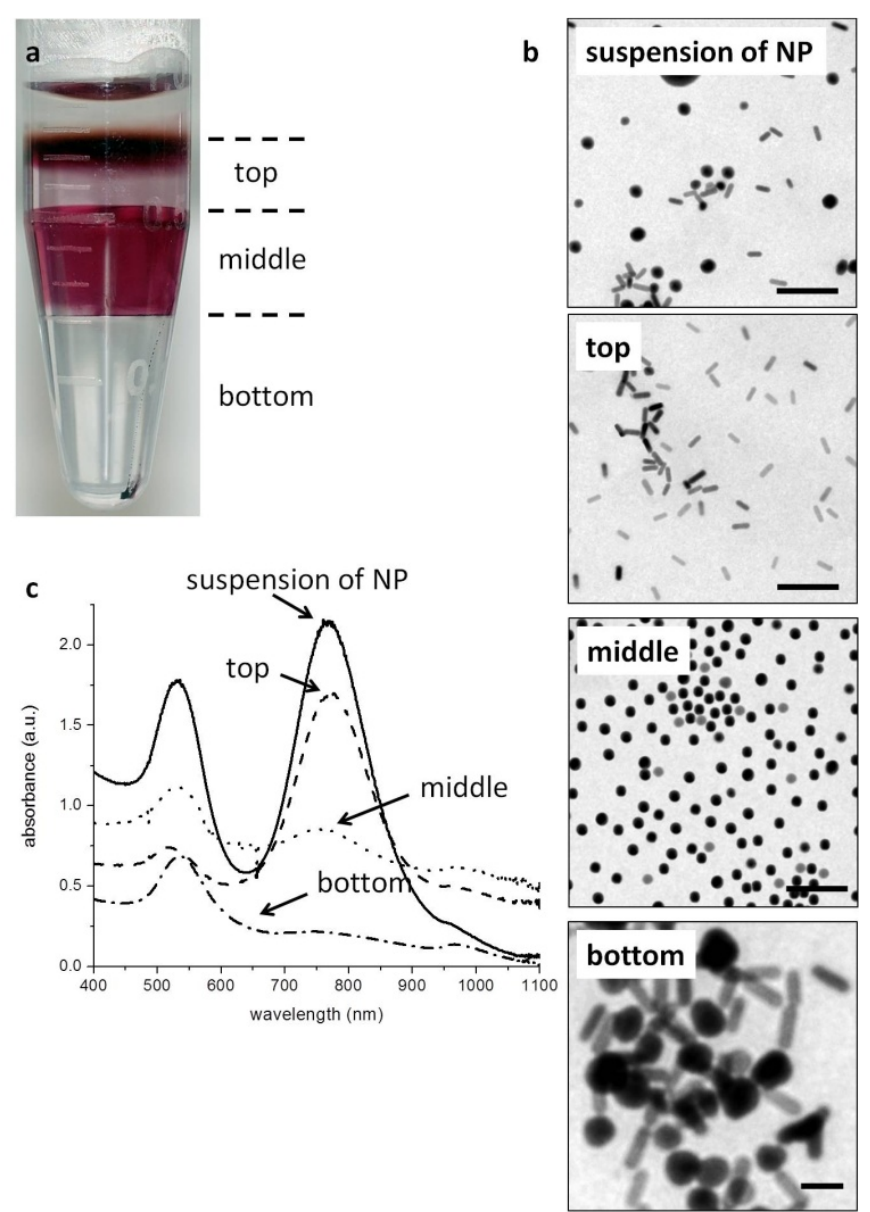
highly shape- and size-dependent. Similarly, the middle phase contained $99 \%$ nanospheres, with average diameters $25 \pm 2 \mathrm{~nm}$, indicating a decrease in polydispersity (Table 1).

In conclusion, we have developed a method, using thermodynamically stable phases of liquids as separation media that offers an improvement for a known technique, rate-zonal centrifugation. The phase-separated media overcome the difficulties associated with the conventional media (e.g., layered systems) such as collection of samples after the enrichment and the lack of stability over time. We expect this stability to be useful to enhance the separation of other objects such as proteins and DNA, where long periods of ultracentrifugation are typically necessary. In addition, the robustness of interfaces of the phase-separated systems allows scaling of the separations such that larger amounts of purified materials can be obtained by using bigger containers. 
Table 1. Quantification of separation of nanoparticles using an aqueous three-phase system (MuPS). We include the densities $\left(\rho, \mathrm{g} / \mathrm{cm}^{3}\right)$ and viscosities $(\eta, \mathrm{cP})$ of each phase of this threephase system composed of Brij 35 (26\% w/v), PEOZ (30\% w/v) and Ficoll (35\% w/v). We designated entries whose counts of particles were less than five per field of view N.A. (not applicable).

\begin{tabular}{cccccccc}
\hline $\begin{array}{c}\text { Layer of } \\
\text { MuPS }\end{array}$ & $\begin{array}{c}\boldsymbol{\rho} \\
\left(\mathbf{g} / \mathbf{c m}^{\mathbf{3}}\right)\end{array}$ & $\begin{array}{c}\boldsymbol{\eta} \\
\mathbf{( c P )}\end{array}$ & $\boldsymbol{\Theta}_{\boldsymbol{N} \boldsymbol{S}^{\boldsymbol{a}}}^{\boldsymbol{c}}$ & $\boldsymbol{\Theta}_{\boldsymbol{N R}^{\boldsymbol{b}}}^{\boldsymbol{c}}$ & $\begin{array}{c}\text { NS Diameter } \\
(\mathbf{n m})\end{array}$ & $\begin{array}{c}\text { NR Length } \\
(\mathbf{n m})\end{array}$ & $\begin{array}{c}\text { NR Thickness } \\
(\mathbf{n m})\end{array}$ \\
\hline top & 1.031 & 30.8 & 0.008 & 0.991 & N.A. & $36 \pm 4$ & $11 \pm 1$ \\
middle & 1.045 & 541.9 & 0.992 & 0.007 & $25 \pm 2$ & N.A. & N.A. \\
bottom & 1.112 & 139.0 & 0.4 & 0.6 & $244 \pm 36$ & $289 \pm 27$ & $100 \pm 9$ \\
\hline
\end{tabular}

${ }^{\text {a }} \mathrm{NS}$ is the abbreviation for nanospheres.

${ }^{\mathrm{b}} \mathrm{NR}$ is the abbreviation for nanorods.

${ }^{\mathrm{c}}$ The number fraction of nanoparticles $(\boldsymbol{\Theta})$, which is defined as the ratio of the counts of number of nanoparticles of certain shape to the total count of nanoparticles in each layer. 


\section{ASSOCIATED CONTENT}

\section{Supporting information}

Characterization methods, pictures of time-dependent penetration of nanoparticles into other aqueous MuPSs under centrifugation, tables of viscosity and density of solutions of polymers and surfactants, and viscosities of different solutions with similar density. This material is available free of charge via the Internet at http://pubs.acs.org.

\section{ACKNOWLEDGMENTS}

This work was supported by the Bill and Melinda Gates Foundation (award number OPP1016360) and by a subcontract from a Department of Energy award to Northwestern University (DE-SC0000989).This work was performed in part using the facilities of the Center for Nanoscale Systems (CNS), a member of the National Nanotechnology Infrastructure Network (NNIN), which is supported by the National Science Foundation under NSF (award number ECS-0335765). CNS is part of the Faculty of Arts and Sciences at Harvard University. O.A. thanks Professor Osman Bakr of KAUST for fruitful discussions. R.V.M. acknowledges funding by the FP7 People program under the project Marie Curie IOF-275148. A. A. K. acknowledges financial support from the Office of Naval Research through the NDSEG fellowship program. The authors thank Professor David Weitz and the Weitz group for the use of their rheometry facilities, and Ms. Felice Frankel for her valuable advice on photography.

\section{RERERENCES}

1. Jiang, W.; Kim, B. Y. S.; Rutka, J. T.; Chan, W. C. W. Nature Nanotech. 2008, 3, 145150.

2. $\quad$ Narayanan, R.; El-Sayed, M. Nano Lett. 2004, 4, 1343-1348.

3. Murphy, C. J.; Sau, T. K.; Gole, A. M.; Orendorff, C. J.; Jinxin Gao; Gou, L.; Hunyadi, S. E.; Li, T. J. Phys. Chem. B 2005, 109, 13857-13870.

4. Adair, J. H.; Suvaci, E. Curr. Opin. Colloid In. 2000, 5, 160-167.

5. Lee, K.-S.; El-Sayed, M. A. J. Phys. Chem. B 2005, 109, 20331-20338. 
6. Alekseeva, A. V.; Bogatyrev, V. A.; Dykman, L. A.; Khlebtsov, B. N.; Trachuk, L. A.; Melnikov, A. G.; Khlebtsov, N. G. Appl. Optics 2005, 44, 6285-6295.

7. Jain, P. K.; Lee, K. S.; El-Sayed, I. H.; El-Sayed, M. A. J. Phys. Chem. B 2006, 110, $7238-7248$.

8. Huang, X.; Neretina, S.; El-Sayed, M. A. Adv. Mater. 2009, 21, 4880-4910.

9. Jana, N. R.; Gearheart, L.; Murphy, C. J. Adv. Mater. 2001, 13, 1389-1393.

10. Sharma, V.; Park, K.; Srinivasarao, M. Proc. Natl. Acad. Sci. 2009, 106, 4981-4985.

11. Khanal, B. P.; Zubarev, E. R. J. Am. Chem. Soc. 2008, 130, 12634-12635.

12. Kim, F.; Song, J. H.; Yang, P. J. Am. Chem. Soc. 2002, 124, 14316-14317.

13. Wei, G.-T.; Liu, F.-K.; Wang, C. R. C. Anal. Chem. 1999, 71, 2085-2091.

14. Hanauer, M.; Pierrat, S.; Zins, I.; Lotz, A.; Sonnichsen, C. Nano Lett. 2007, 7, 28812885.

15. Xiong, B.; Cheng, J.; Qiao, Y.; Zhou, R.; He, Y.; Yeung, E. S. J. Chromatogr. A 2011, 1218, 3823-3829.

16. Bai, L.; Ma, X.; Liu, J.; Sun, X.; Zhao, D.; Evans, D. G. J. Am. Chem. Soc. 2010, 132, 2333-2337.

17. Sun, X.; Tabakman, S. M.; Seo, W.-S.; Zhang, L.; Zhang, G.; Sarah Sherlock; Bai, L.;

Da, H. Angew. Chem. Int. Ed. 2009, 48, 939-942.

18. Qiu, P.; Mao, C. Adv. Mater. 2011, 23, 4880-4885.

19. Chen, G.; Wang, Y.; Tan, L. H.; Yang, M.; Tan, L. S.; Chen, Y.; Chen, H. J. Am. Chem. Soc. 2009, 131, 4218-4219.

20. Tyler, T. P.; Henry, A.-I.; Duyne, R. P. V.; Hersam, M. C. J. Phys. Chem. Lett. 2011, 2, 218-222.

21. Mace, C. R.; Akbulut, O.; Kumar, A. A.; Shapiro, N. D.; Derda, R.; Patton, M. R.;

Whitesides, G. M. J. Am. Chem. Soc. [Online early access]. DOI:10.1021/ja303183z. Published Online: May 17, 2012

22. Nikoobakht, B.; El-Sayed, M. A. Chem. Mater. 2003, 15, 1957-1962.

23. Jana, N. R.; Gearheart, L.; Murphy, C. J. J. Phys. Chem. B 2001, 105, 4065-4067.

24. Jain, P. K.; Lee, K. S.; El-Sayed, I. H.; El-Sayed, M. A. J. Phys. Chem. B 2006, 110, 7238-7248. 